\title{
Infusion Therapy AND Transfusion Medicine: Quo vadis?
}

Three years ago, the editorial board of INFUSIONSTHERAPIE UND TRANSFUSIONSMEDIZIN decided to redefine the concept of the journal: Steps were taken to enhance the journal's acceptance on the international level, to emphasize the significance of transfusion medicine as a clinical discipline, and to raise the scientific quality of published work. Thus, the journal's title changed to INFUSION THERAPY AND TRANSFUSION MEDICINE, and authors wishing to contribute a paper were asked to submit the manuscript in English. 'Infusion therapy' remains an essential part of the journal's title, indicating that hemotherapeutic relevance of published data is a major concern of the editorial policy. Manuscripts submitted to INFUSION THERAPY AND TRANSFUSION MEDICINE are subjected to a review process by at least two expert referees, ensuring scientific quality of accepted manuscripts.

The modified concept of the journal has been widely accepted: Readers reflect a broad spectrum of clinical disciplines, and subscriptions to INFUSION THERAPY AND TRANSFUSION MEDICINE raise continuously. However, the journal's impact as represented by the impact factor has persistently been a matter of concern among members of our society. On the one hand, we observe that the impact factor of INFUSION THERAPY AND Transfusion MEdicine stabilizes at a level similar to that of other well-known and accepted journals of specialist societies such as the Schweizer Medizinische Wochenschrift or the Internist. On the other hand, it must be allowed to ask critically whether a tool like the impact factor appropriately reflects the significance of Infusion Therapy and TRANSFUSION MEDICINE, and to take into account the very special development of transfusion medicine in the context of clinical, scientific and legal demands.

Transfusion medicine in the beginning of its development as an independent discipline has mainly been concerned with practical items of collection, storage and clinical use of blood products. Scientific aspects of transfusion medicine during that time were oriented toward aspects of application of blood components in the clinical context. They were strongly influenced by national instructions to ensure safe blood transfusions, and their results therefore had relevance mostly in the context of these national structures. Over the last 15 years, however, innovative techniques adopted from basic research were brought into the daily routine of work in transfusion medicine, helping to establish a more fundamental scientific potential in this field and providing the opportunity to publish results in international 'high-impact' journals.

Nevertheless, despite of a raising reputation of German transfusion medicine within the international scientific community, the significance of a national periodical in transfusion medicine should not be underestimated. There is a demand for a scientific medium allowing to publish application-oriented scientific aspects of transfusion medicine within the context of national legal rules. The transfusion act requires to define, to maintain, and to communicate the state of medical science and technology concerning the use of blood products. Since the state of medical science and technology is a dynamic one, fulfilling such legal demands requires in many respects a high degree of flexibility of medical facilities. One of the outstanding aims of INFUSION THERAPY AND TRANSFUSION MEDICINE is therefore to inform rapidly, reliably and with competence about new developments, helping to comply with legal demands in daily work.

The other essential interest of INFUSION THERAPY AND TRANSFUSION MEDICINE is to provide a forum for aspects of clinical transfusion medicine: To ensure its future as an independent discipline, transfusion medicine has to cover both aspects, that of fundamental research and that of clinical research. It has to define and to sharpen its own scientific profile in fundamental research on the one hand, and it has to promote its interdisciplinary character in clinical research on the other hand. Both aspects form an essential part of the identity of modern trans- 
fusion medicine and should be tightly linked. The combination of a unique scientific profile in basic research and close interdisciplinary cooperations in clinical research will ensure survival of transfusion medicine as an independent medical speciality. To represent transfusion medicine in its complexity and clinical relevance, the young generation in this discipline has to prove competence in fundamental research and in applied research in the clinical setting equally. INFUSION THERAPY AND TRANSFUSION MEDiCINE provides excellent opportuni- ties for clinical transfusion medicine to qualify itself at a high level in an internationally accepted way.

The editorial board therefore is confident that INFUSION Therapy And Transfusion Medicine will be intensively used as the appropriate forum to discuss scientific aspects of clinical transfusion medicine and will essentially contribute to ensure the future of clinical transfusion medicine.

The Editors 